\title{
Mitochondria-mediated tumstatin peptide-induced HepG2 cell apoptosis
}

\author{
YAN LIU ${ }^{1}$, JIHONG LI ${ }^{1}$, HUI XU ${ }^{1}$, YUWEN ZHANG ${ }^{1}$, YUANLI LIU ${ }^{1}$ and XINGHAN LIU ${ }^{1,2}$ \\ ${ }^{1}$ Department of Biochemistry and Molecular Biology, ${ }^{2}$ Laboratory of Medical Genetics, Harbin Medical University, \\ The Key Laboratory of Biomedical Engineering of Heilongjiang Province, Harbin 150081, P.R. China
}

Received July 10, 2009; Accepted August 21, 2009

\section{DOI: 10.3892/ijmm_00000276}

\begin{abstract}
The effect of a 19-amino-acid C-terminal peptide of tumstatin (aa 185-203, peptide 19) on human hepatoma cell (HepG2) proliferation was studied, as well as the mechanism by which it induces tumor cell apoptosis. Recombinant peptide 19 was purified by chitin affinity chromatography and identified by Tricine-SDS-PAGE. The DTT was removed with sephadex G-10. MTT colorimetry was used to evaluate the proliferation of tumor cells. Hematoxylin and eosin staining (H\&E staining) and $\mathrm{AO} / \mathrm{EB}$ double staining were used to view morphological changes during apoptosis. Mitochondrial potential was measured via flow cytometer. Western blot analysis was performed to detect the transfer of cytochrome $\mathrm{C}$ from mitochondria to the cytoplasm and to monitor the expression levels of caspase-8, caspase-9, Fas, p53, Bcl-2, Bax and Bid in human hepatoma cells. Recombinant peptide 19 effectively suppressed the proliferation of HepG2 cells and induced apoptosis. Each of the two effects had a dosedependent relationship with recombinant peptide 19. Peptide 19 upregulated the expression of caspase-9, Fas, p53, Bax and Bid, downregulated the expression of Bcl-2 and had little effect on the expression of caspase 8. Peptide 19 decreased the mitochondrial membrane potential and induced the release of cytochrome $\mathrm{C}$ from mitochondria to the cyto-plasm. In conclusion, peptide 19 induced HepG2 cell apoptosis through the mitochondrial apoptosis pathway.
\end{abstract}

\section{Introduction}

Hepatocellular carcinoma (HCC) is one of the five highestmortality tumors in the world and its incidence rate is currently increasing. The most important task in treatment of this cancer is to effectively kill hepatoma cells or induce their apoptosis. Currently, the most widely used therapies for hepatoma are chemotherapy and radiotherapy, which usually have severe side effects. Therefore, the development of an efficacious and

Correspondence to: Dr Xinghan Liu, Department of Biochemistry and Molecular Biology, Harbin Medical University, The Key Laboratory of Biomedical Engineering of Heilongjiang Province, Harbin 150081, P.R. China

E-mail: hydlxh@hotmail.com

Key words: tumstatin, apoptosis, mitochondrial pathway low toxicity anti-hepatoma drug is an attractive study area. New peptide drugs may have these characteristics, including some tumor suppressor proteins such as apoptin, endostatin and tumstatin. Identifying the core effective area of these tumor suppressor proteins could result in purer anti-tumor drugs.

Tumstatin is one part of the non-collagen region of the $\alpha_{3}$ chain of collagen IV. It was discovered as an antigen of Goodpasture syndrome (1). Tumstatin consists of the NC1 domain and the twelve amino acids at the $\mathrm{C}$ terminus of three helix domains that are in the middle of the $\alpha_{3}$ chain of collagen IV (2). Tumstatin has 244 amino acids and its gene has $738 \mathrm{bp}$. The molecular weight of tumstatin is $\sim 28 \mathrm{kDa}$. Previously, some studies have indicated that the $\alpha_{1}$ chain and the $\alpha_{2}$ chain of collagen IV also have anti-tumor properties and their active sequences are arrestin and canstatin, respectively $(3,4)$.

In 2000 , Kamphaus et al $(3,4)$ found that tumstatin had two different active anti-tumor areas. One is a peptide of 19 amino acids (185-203 amino acids) near the $\mathrm{C}$ terminus that has the direct anti-tumor effect of suppressing tumor cell proliferation and inducing tumor cell apoptosis. The other is a peptide of seven amino acids (74-98 amino acids) near $\mathrm{N}$ terminus that has an indirect anti-tumor effect of suppressing tumor angiogenesis $(2,5,6)$. Tumstatin is a very effective angiogenesis suppressor, with activity ten times greater than that of endostatin. Tumstatin combines with $\alpha \mathrm{v} \beta_{3}$ to suppress the activity of FAK, PI3 kinase and PKB and thus blocking the integrin-induced CAP-dependent protein synthesis pathway and selectively suppressing endothelial cell proliferation. Therefore, tumstatin has a strong anti-angiogenesis effect (2,7-9). Previously, it has been proven in a mouse melanoma model that peptide 19 of tumstatin suppresses tumor growth in a conformation-dependent manner. A short peptide of seven amino acids (CNYYSNS) from the $\mathrm{N}$ terminus (185-191 amino acids) of tumstatin has stronger anti-tumor activity. The three-dimensional structures of the CNYYSNS peptide and peptide 19 of tumstatin show that the YSNS sequence has a $\beta_{2}$ angle conformation. Further structural studies showed that this $\beta_{2}$ angle is necessary for bioactivity. These peptides suppress melanoma growth effectively and selectively by combining with $\alpha v \beta_{3}$ integrin (10). Therefore, tumstatin has become more attractive as an anti-tumor therapy. In our previous study, peptide 19 of tumstatin was proven to be able to suppress tumor cell proliferation and induce tumor cell apoptosis (11). However, 
the mechanism of peptide 19-induced tumor cell apoptosis was still not clear. This study identified the role of the mitochondrial apoptosis pathway in peptide 19-induced hepatocellular carcinoma cell apoptosis.

\section{Materials and methods}

Reagents. RPMI-1640 cell culture medium (Gibco), fetal calf serum (PAA) and trypsin (DIFCO) were purchased from Invitrogen (USA). DMSO, MTT and sephadex G-10 medium (Pharmacia) was purchased from Sigma. A chitin affinity chromatography column was purchased from NEB. Acridine orange (AO) was from AMRESCO. A live cell mitochondrial membrane potential fluorescence assay kit was purchased from Keygen. Anti-caspase- 8 antibody, anti-caspase- 9 antibody, anti-cytochrome $\mathrm{C}$ antibody, anti-p53 antibody, anti- $\beta$-actin antibody, anti-Bcl-2 antibody, anti-Bax antibody, anti-Bid antibody, anti-Fas antibody and secondary antibody were purchased from Santa Cruz.

Cell culture. The human hepatoma cell line HepG2 (purchased from Shanghai Institute of Cell Biology) was cultured in RPMI-1640 culture medium with $10 \%$ fetal calf serum and incubated with $5 \% \mathrm{CO}_{2}$ at $37^{\circ} \mathrm{C}$. Cells were grown on plates and were subcultured after $0.25 \%$ trypsin treatment. The experiments were performed when the ratio of cell fusion reached $70 \%$.

Purification of recombinant peptide 19 of tumstatin. We constructed plasmid pTYB2-T19 to produce peptide 19 previously. It was purified following the method of Wang et al (11). The DTT was removed with sephadex G-10. Finally, peptide 19 was identified by Tricine-SDS-PAGE.

Detection of HepG2 proliferation. HepG2 cells were adjusted to a concentration of $5 \times 10^{4} / \mathrm{ml}$ by using a cell counter. A total of $200 \mu 1$ cell suspension was added into each well of 96-well plates. Recombinant peptide 19 was applied at various final concentrations, including $0,40,80,120,160$ and $200 \mu \mathrm{g} / \mathrm{ml}$ after the cells were cultured for $24 \mathrm{~h}$ and adhered to the plates. Forty-eight hours after peptide 19 treatment, the culture medium with peptide 19 was removed, the cells were washed with PBS three times and $200 \mu 1$ of new culture medium mixed with $20 \mu 1 \mathrm{MTT}$ was added to each well. Four hours later, the supernatant was removed and $150 \mu \mathrm{l}$ DMSO was added to each well. The plates were then shaken for $10 \mathrm{~min}$ to dissolve the crystals. The absorbance of each well was detected by using an enzyme label analyzer (Bio-Rad) at $490 \mathrm{~nm}$. Then a curve was constructed with the drug concentration as the $\mathrm{X}$ axis and the absorbance (\%) as the $\mathrm{Y}$ axis.

\section{Detection of HepG2 apoptosis}

$H \& E$ staining. HepG2 cells grown to log phase were digested with $0.25 \%$ trypsin. A cell suspension with $5 \times 10^{4}$ cells was added to each well. The cells were cultured with pretreated coverslips for another $24 \mathrm{~h}$ in $24-w e l l$ plates. The cells were divided into two groups. The peptide 19-treated group was mixed with peptide 19 at a final concentration of $40 \mu \mathrm{g} / \mathrm{ml}$. The other group was the control group and it was mixed with the same volume of PBS. At the end of the $24 \mathrm{~h}$, all the coverslips were picked out and fixed with acetone for $10 \mathrm{~min}$, washed with PBS three times and stained with hematoxylin for $3 \mathrm{~min}$. The coverslips became blue after they were washed with distilled water. The coverslips were put into eosin for 2 min, washed with water and treated with $30 \%$ alcohol and then $100 \%$ alcohol for $1 \mathrm{~min}$. Xylene transparence was performed on these coverslips for $10 \mathrm{~min}$. Each microscope slide was dried by fire. Then, one drop neutral balata was added to each. The coverslips treated with xylene were put onto the neutral balata individually with the cell side down. After drying at $37^{\circ} \mathrm{C}$, the cells were observed under a microscope.

AO/EB fluorescence staining. $\mathrm{AO}$ and $\mathrm{EB}$ were dissolved in $\mathrm{pH}$ 7.2 PBS separately to a final concentration of $100 \mu \mathrm{g} / \mathrm{ml}$. Equal amounts of them were mixed before use. Cell culture, drug application and H\&E staining were similar to the previous experiments. The coverslips were treated with $10 \mu 1 \mathrm{AO} / \mathrm{EB}$ mixture for $1 \mathrm{~min}$ and then the mixture was absorbed with filter paper. A fluorescence microscope (Nikon) was used to observe the cells.

Detection of mitochondrial membrane potential. Based on the method of Desai et al (12), the mitochondrial membrane potential was detected by fluorescence assay kit. The cells were pre-treated with recombinant peptide $19(40 \mu \mathrm{g} / \mathrm{ml})$ for 0,12 , 24 or $36 \mathrm{~h}$ and then washed with PBS two times (centrifuging at $2,000 \mathrm{rpm}$ for $5 \mathrm{~min}$ ). A total of $1 \times 10^{6}-2 \times 10^{6}$ cells were collected for each group. The cells were resuspended in the culture medium with $5 \mu \mathrm{g} / \mathrm{ml} \mathrm{JC}-1$ and incubated for $15 \mathrm{~min}$ in an incubator. Then they were centrifuged again $(2,000 \mathrm{rpm}$, $5 \mathrm{~min}$ ) to remove the supernatant and the pellets were resuspended with $1 \mathrm{ml}$ pre-warmed culture medium. Apoptosis was detected with a FACSCalibur flow cytometer $(\mathrm{Ex}=488 \mathrm{~nm} ; \mathrm{Em}=530 \mathrm{~nm})$.

Western blot analysis to detect the expression of caspase 8, caspase 9, Bcl-2, Bax, Bid, p53 and Fax and the release of cytochrome $C$. Western blot analysis was used to detect the expression of caspase 8, caspase 9, Bcl-2, Bax, Bid, p53 and Fas. HepG2 cells were treated with $40 \mu \mathrm{g} / \mathrm{ml}$ recombinant peptide 19 for 24,36 and $48 \mathrm{~h}$. All the cells were collected by centrifuge ( $800 \mathrm{rpm}, 5 \mathrm{~min}$ ) and washed with cold PBS three times. Then the cells were resuspended in $100 \mu 1$ RIPA buffer and incubated on ice to lyse for $1 \mathrm{~h}$. After that, all the samples were centrifuged at $16,000 \mathrm{rpm}$ at $4{ }^{\circ} \mathrm{C}$ for $10 \mathrm{~min}$ and the supernatant was retained. A nucleic acid and protein analyzer (Amersham Biosciences) was used to detect the protein concentration in the supernatant. The Western blot procedure followed the instructions of the kit. The primary antibodies were diluted as follows: anti-caspase-8 (1:1000, v/v), anticaspase-9 (1:1000, v/v), anti-p53 (1:750, v/v), anti-ß-actin $(1: 1000, \mathrm{v} / \mathrm{v})$, anti-Bcl-2 (1:500, v/v), anti-Bax (1:500, v/v), anti-Bid $(1: 500, \mathrm{v} / \mathrm{v})$, anti-Fas $(1: 500, \mathrm{v} / \mathrm{v})$. The secondary antibody was diluted 1:5000 (v/v).

In order to detect the release of cytochrome $\mathrm{C}$ from mitochondria to the cytoplasm, HepG2 cells were treated with $40 \mu \mathrm{g} / \mathrm{ml}$ recombinant peptide 19 for $24 \mathrm{~h}$ and then washed with PBS. The cell pellet was collected. A total $5 \times 10^{7}$ cells were needed for each extraction. The cells were resuspended in $1.5 \mathrm{ml}$ cold Mito-Cyto Buffer, lysed and centrifuged at 


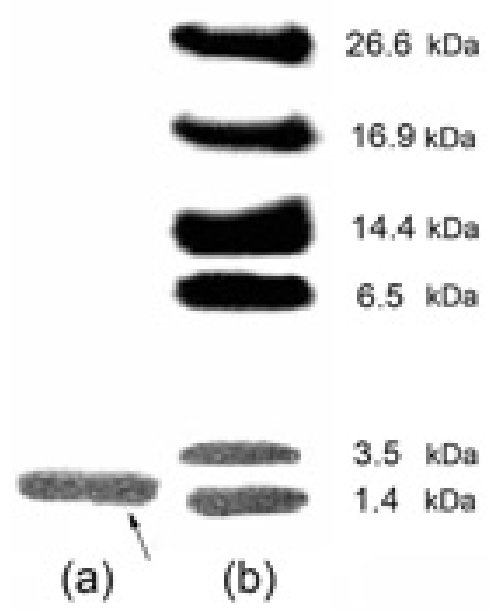

Figure 1. Tricine-SDS-PAGE analysis. (a) Recombinant peptide 19; (b) protein molecular weight markers.

$800 \mathrm{x} \mathrm{g}$ at $4^{\circ} \mathrm{C}$ for $5 \mathrm{~min}$ to save the supernatant. Then the supernatant was centrifuged again at $12,000 \mathrm{x}$ g for $10 \mathrm{~min}$ to separate the mitochondria (in pellet) and the cytoplasm (the supernatant). Mito-Cyto Buffer $(0.2 \mathrm{ml})$ was used to wash the mitochondria and resuspend the mitochondria. The Western blot procedure followed the instructions of the kit. The anticytochrome $\mathrm{C}$ antibody was diluted to 1:500 (v/v) and the secondary antibody was diluted to 1:5000 (v/v).

\section{Results}

Expression and purification of recombinant tumstatin peptide 19. Recombinant peptide 19 of tumstatin was purified by chitin affinity chromatography and DTT was removed with sephadex G-10. Recombinant peptide 19 of tumstatin presented a specific band near $2 \mathrm{kd}$ in Tricine-SDS-PAGE (Fig. 1).

Recombinant peptide 19 from tumstatin inhibits HepG2 cell proliferation. Recombinant peptide 19 of tumstatin was added to HepG2 cell culture medium at various concentrations $(40-200 \mu \mathrm{g} / \mathrm{ml})$ and the cells were grown for another $48 \mathrm{~h}$. The inhibitory effect of peptide 19 on HepG2 proliferation correlated positively with the peptide 19 concentration. The half-maximal inhibitory concentration of recombinant peptide 19

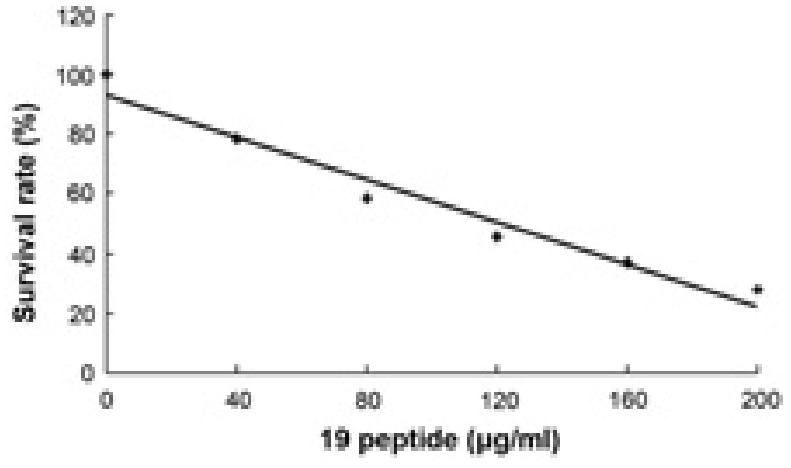

Figure 2. Inhibition of HepG2 cell proliferation by peptide 19.

on $\mathrm{HepG} 2$ growth was $122.7 \mu \mathrm{g} / \mathrm{ml}\left(\mathrm{IC}_{50}=122.7 \mu \mathrm{g} / \mathrm{ml}\right)$ based on linear regression analysis (Fig. 2).

\section{Recombinant peptide 19 from tumstatin induces HepG2 apoptosis}

$H \& E$ staining. After treatment with recombinant peptide 19 of tumstatin $(80 \mu \mathrm{g} / \mathrm{ml})$ for $24 \mathrm{~h}$, HepG2 cells displayed more apoptotic characteristics under H\&E staining. Significant apoptotic morphological changes were observed, such as condensed nuclei, nuclear lysis and membrane shrinkage (Fig. 3).

AO/EB fluorescence staining. After treatment with recombinant peptide 19, AO/EB fluorescence staining of HepG2 cells revealed a uniform condensed round clump structure typical of early apoptotic cells and the condensed orange chromatin typical of late apoptotic cells (Fig. 4).

The mechanism for recombinant peptide 19-induced HepG2 apoptosis

Mitochondrial membrane potential. A decrease in mitochondrial membrane potential occurred at the early stage of apoptosis. The change in mitochondrial membrane potential of recombinant peptide 19-treated HepG2 cells was detected by flow cytometry based on JC-1 fluorescent staining. HepG2 cells were treated with recombinant peptide 19 for 12,24 or $36 \mathrm{~h}$. The increase in their fluorescence signal was 8.0, 25.3 and $68.6 \%$, respectively, indicating that their mitochondrial membrane potential decreased with longer treatment (Fig. 5).

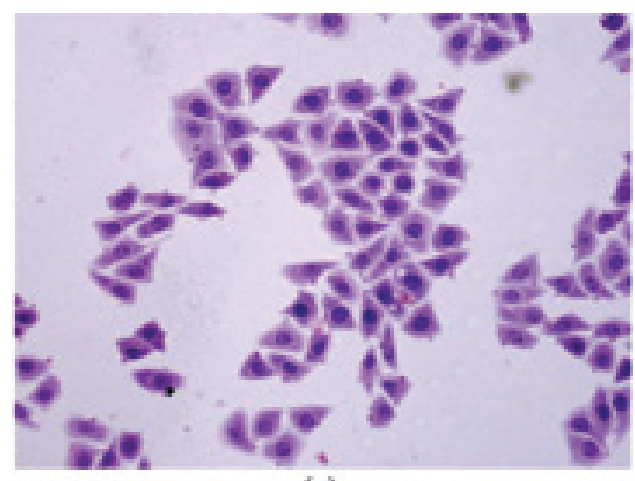

(a)

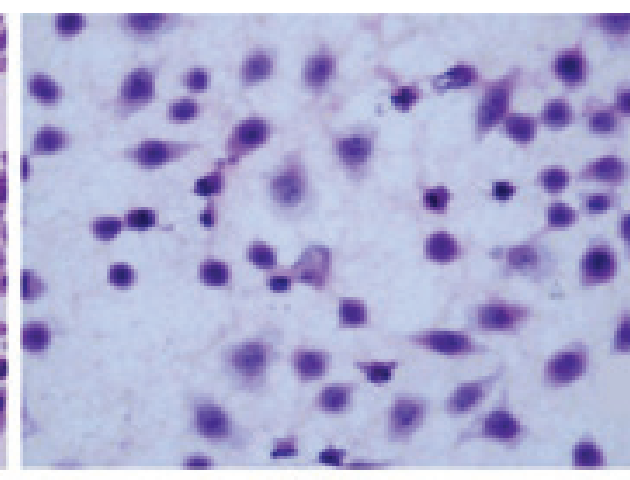

(b)

Figure 3. Effect of peptide 19 on HepG2 cells (H\&E staining). (a) Control; (b) $80 \mu \mathrm{g} / \mathrm{ml}$ peptide 19. 


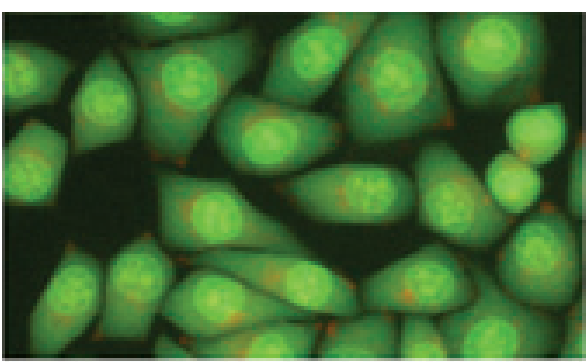

(a)

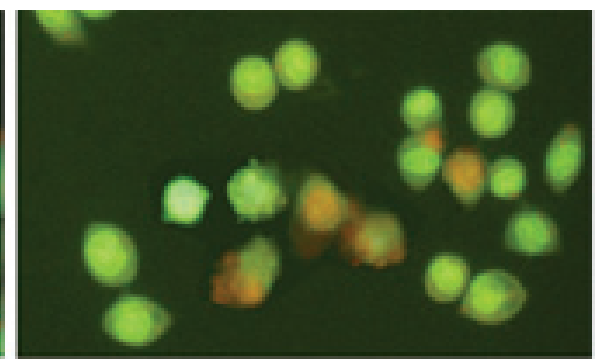

(b)

Figure 4. Effect of peptide 19 on HepG2 cells (AO/EB staining). (a) Control; (b) $80 \mu \mathrm{g} / \mathrm{ml}$ peptide 19.

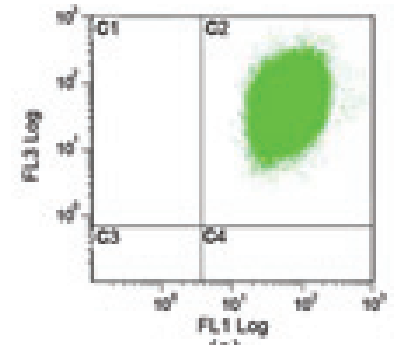

(a)

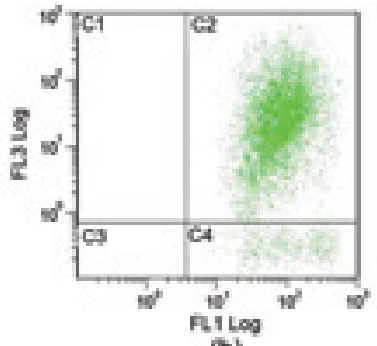

(b)

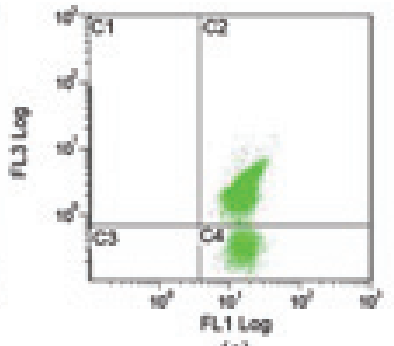

(c)

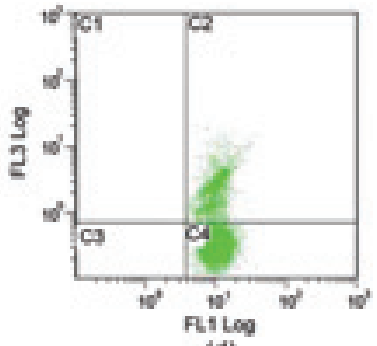

(d)

Figure 5. Effect of peptide 19 on mitochondrial membrane potential. (a) Control; (b) peptide 19 for $12 \mathrm{~h}$; (c) peptide 19 for 24 h; (d) peptide 19 for $36 \mathrm{~h}$.
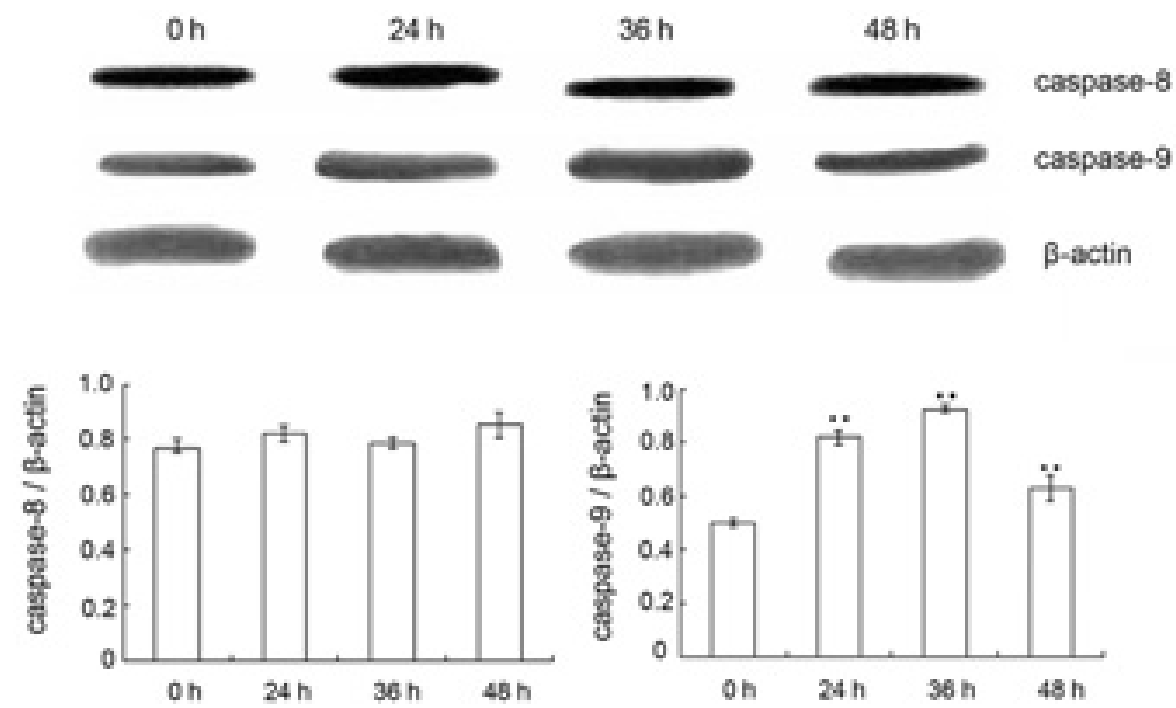

Figure 6. Effect of peptide 19 on the levels of caspase 8 and caspase 9 (Western blot analysis). HepG2 cells were treated with peptide 19 for $0,24,36$ and $48 \mathrm{~h}$.

Expression levels of caspase 8, caspase 9, p53, Fas, Bcl-2, Bax and Bid. The levels of caspase 8, caspase 9, p53, Fas, Bcl-2, Bax and Bid were detected by Western blot analysis. Along with the increasing time of exposure to recombinant peptide 19, the expression of Bcl-2 decreased, the expression of caspase 9, Bax and Bid increased and the ratio of Bcl-2/ Bax decreased. The expression of caspase 8 change little after treatment with recombinant peptide 19 (Figs. 6 and 7). The expression of p53 and Fas increased (Fig. 8).

Detection of the release of cytochrome C. During apoptosis based on the mitochondrial pathway, cytochrome $\mathrm{C}$ in the mitochondria is released into the cytoplasm. Western blot analysis indicated that cytochrome $\mathrm{C}$ in the HepG2 control group localized to the mitochondria, while there was no cytochrome C in the cytoplasm. After HepG2 cells were treated with recombinant peptide 19 of tumstatin for $24 \mathrm{~h}$, cytochrome $\mathrm{C}$ was seen in the cytoplasm, while the amount of it in mitochondria went down. This indicated that cytochrome $\mathrm{C}$ was released from the mitochondria into the cytoplasm (Fig. 9).

\section{Discussion}

Tumstatin was first found as a self-antigen of Goodpasture syndrome. It comes from the non-collagen area of the $\alpha_{3}$ chain 

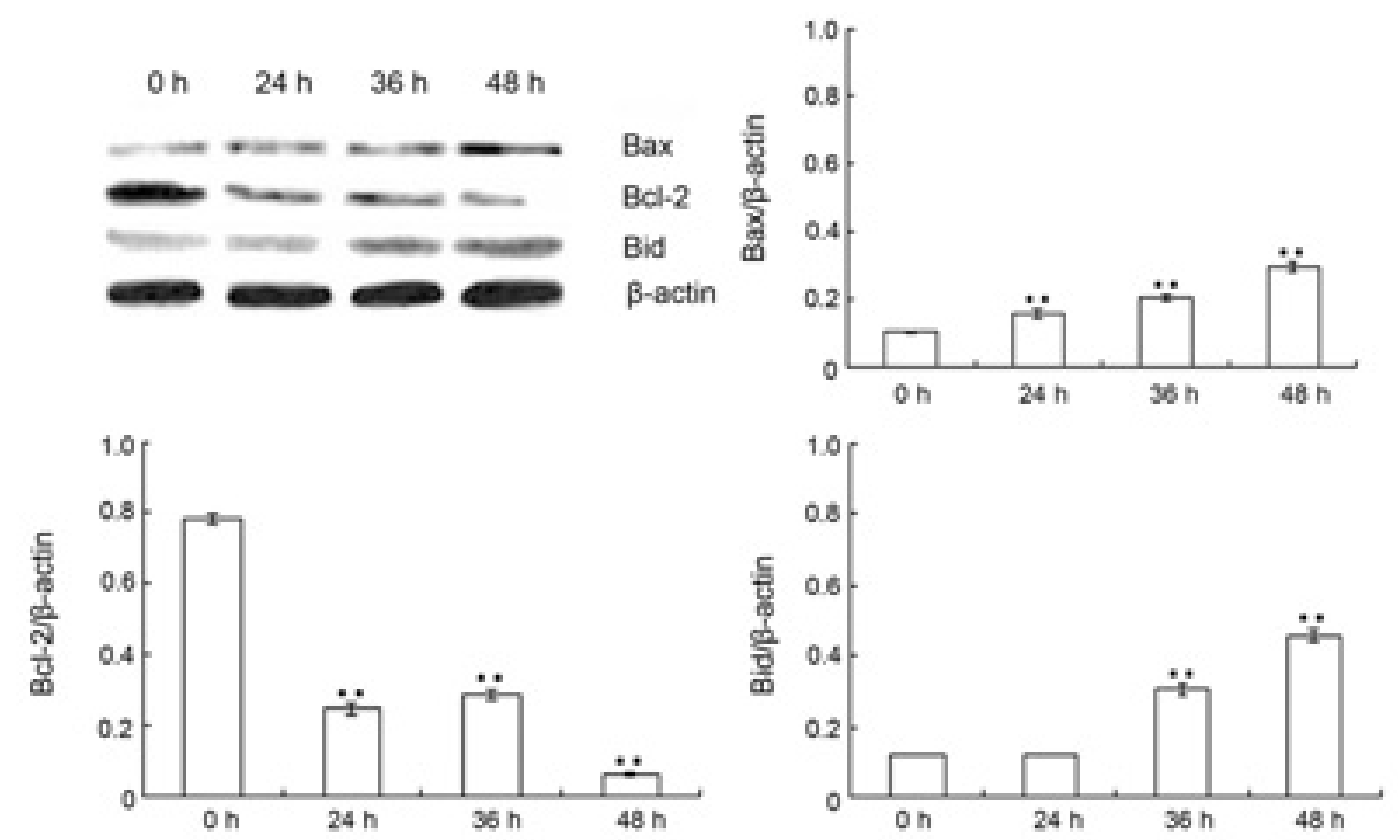

Figure 7. Effect of peptide 19 on the levels of Bax, Bcl-2 and Bid (Western blot analysis). HepG2 cells were treated with peptide 19 for 0, 24, 36 and 48 h.
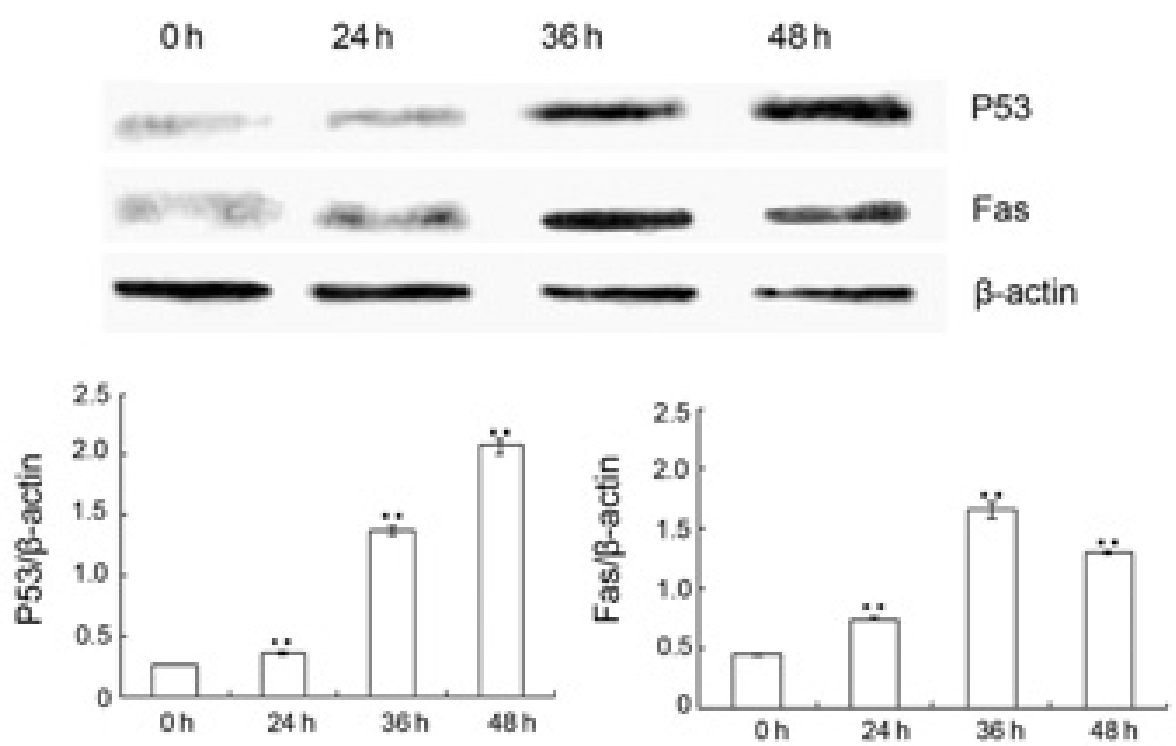

Figure 8. Effect of peptide 19 on the levels of p53 and Fas (Western blot analysis). HepG2 cells were treated with peptide 19 for 0, 24, 36 and 48 h.

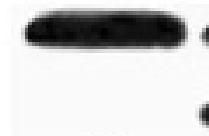

(a)

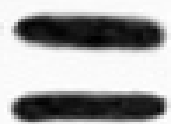

(b)
Cylosolic fraction

Mitochondria fraction

Figure 9. The distribution of cytochrome C (Western blot analysis). HepG2 cells were treated with peptide 19 for 24 h. (a) Control; (b) peptide 19.

of collagen IV. The study of Kamphaus et al (4) indicates that peptide 19 of tumstatin (185-203 amino acids) suppresses tumor cell proliferation and promotes tumor cell apoptosis. Our previous study also showed that peptide 19 of tumstatin indeed promoted tumor cell apoptosis. However, its mechanism remained unknown.
It is believed that there are three apoptosis pathways. The first is a membrane signaling pathway, the second is a mitochondrial signaling pathway and the third is an endoplasmic reticulum signaling pathway. The focus of most studies is the mitochondrial signaling pathway.

In the mitochondrial pathway of apoptosis, a decrease in mitochondrial membrane potential and the release of cytochrome $\mathrm{C}$ are believed to be early steps in the process of apoptosis (13). These occur before the activation of caspase 9 and nuclear apoptosis. Once this crisis of mitochondrial membrane potential has occurred, apoptosis has become irreversible. Some lipophilic cation fluorescent dyes can accumulate in the plasma of mitochondria depending on their membrane potential. The fluorescence intensity represents the electronegative intensity of the mitochondrion inner membrane. 
In this study, we found that recombinant peptide 19 of tumstatin induced a change in HepG2 cell mitochondrial membrane potential change by $\mathrm{JC}-1$ staining and flow cytometric detection. Three groups of HepG2 cells were incubated with a concentration of peptide $1975 \%$ of the $\mathrm{IC}_{50}$ for 12,24 and $36 \mathrm{~h}$, respectively. In the normal cells, the green fluorescence accumulated in the $\mathrm{C} 2$ area, while the green fluorescence transferred from the $\mathrm{C} 2$ area to the $\mathrm{C} 4$ area in apoptotic cells and the intensity of green fluorescence was positively correlated with the severity of the apoptosis. In our study, the percentage of green fluorescence transferred to the C4 area was $8.0,25.3$ and $68.6 \%$ for the three incubation time groups, respectively. This indicated that mitochondrial membrane potential decreased with increasing peptide 19 incubation times. Under normal conditions, cytochrome C settles among phospholipids along the mitochondrial inner membrane. During apoptosis, cytochrome $\mathrm{C}$ is released from mitochondria to the cytoplasm and forms the apoptosome together with Apaf-1, pro-caspase 9 and ATP/dATP. The activated caspase 9 in the apoptosome activates caspase 3, which starts the caspase cascade and induces cell apoptosis (14). By detecting the concentration of cytochrome $C$ in the mitochondria and cytoplasm by Western blot analysis, we found that cytochrome $\mathrm{C}$ only present in the mitochondria and not in the cytoplasm. In the recombinant peptide 19 treatment group, the amount of cytochrome C was similar in the mitochondria and the cytoplasm. The protein level of cytochrome $\mathrm{C}$ in the treatment group was lower than that in the control group. The change in mitochondrial membrane potential and the release of cytochrome $\mathrm{C}$ proved that peptide 19 of tumstatin induced tumor cell apoptosis through the mitochondrial pathway.

The release of cytochrome $\mathrm{C}$ from mitochondria activates the caspase family, especially caspase 9 , which plays a starting role $(15,16)$. By Western blot analysis, it was found that the expression of caspase 8 change little under peptide 19 treatment, however the expression of caspase 9 was significantly upregulated. This suggested that peptide 19 of tumstatin induced tumor cell apoptosis by the mitochondrial pathway.

The Bcl-2 family is the central regulator of proliferation and apoptosis. Bcl-2 family members can form homologous or heterologous dimers. Certain protein dimers may act as a molecular switch in the cell death pathway. Bcl-2 can form a dimer with Bax that can promote cell apoptosis. If the amount of Bax higher than that of Bcl-2, it will promote cell death. Otherwise, it will inhibit cell apoptosis. Activated Bax can increase the permeability of the mitochondrial outer membrane and this will induce the release of cytochrome $\mathrm{C}$. $\mathrm{Bid}$ is a protein promoting apoptosis and the dislocation and insertion of Bax depends on Bid. It is the conformational change of Bax that regulates cell apoptosis. Bid can also combine with Bcl-2 which has the anti-apoptosis function. This dimer blocks the oligomerization of Bax and this enhances the Bax-induced damage to mitochondria (17-19). In this study, $24 \mathrm{~h}$ after peptide 19 treatment of HepG 2 cells, the levels of Bax significantly increased, the expression of Bcl-2 significantly decreased, the ratio of Bcl-2/Bax decreased and the expression of Bid increased. These results indicated that peptide 19 of tumstatin induced cell apoptosis by influencing the expression of Bcl-2 family members, including Bax, Bcl-2 and Bid and thereby activating the mitochondrial pathway.

We also studied the expression of p53 and Fas, since the expression of Bcl-2 was downregulated and the expression of Bax and Bid were upregulated. The key role of p53 in apoptosis has been widely investigated and it can initiate apoptosis mainly by upregulating the expression of apoptosis promoters and downregulating the expression of apoptosis inhibitors (20). p53 can directly combine with the anti-apoptosis protein Bcl-2 outside the nucleus, hence it may inhibit the function of $\mathrm{Bcl}-2$ and directly promote $\mathrm{Bax}$ in the cytoplasm to localize to mitochondria, inducing the release of cytochrome $\mathrm{C}$ and starting the caspase cascade. p53 can increase the amount of Fas on the cell membrane by promoting the transfer of Fas from the Golgi apparatus to the cell membrane. The intracellular death domain of Fas interacts with the death domain at the $\mathrm{C}$ terminus of FADD. FADD accumulates in the cytoplasm and caspase 8 is then activated. The activated caspase 8 can cleave Bid in the cytoplasm. The $\mathrm{C}$ terminal fragment of this cleavage, $\mathrm{tBid}$, is transferred onto the mitochondrial membrane, thus altering its permeability (21). The cleavage of Bid connects the mitochondrial pathway with the death receptor pathway, thus amplifying the apoptotic signal. It was found that peptide 19 of tumstatin upregulates the expression of Fas and $\mathrm{p} 53$. This suggests that the original target during peptide 19 of tumstatin-induced tumor cell apoptosis might be p53. With the upregulated expression of p53, apoptosis-promoting genes such as Bax were activated, the expression of Bcl-2 was inhibited and the Bax/Bcl-2 ratio was enhanced, thus the mitochondrial pathway of apoptosis was initiated. In this study, the upregulated expression of Fas was observed, however there was little influence on the expression of caspase 8 . This indicates that the upregulation of Fas expression was not promoting the death receptor apoptotic pathway. It has been speculated that the upregulation of Fas expression is controlled by p53. Fas might activate caspase 8 , cleave Bid and change mitochondrial membrane permeability in order to change the expression of the Bcl-2 family and enhance apoptosis through the mitochondrial pathway.

Our data showed that peptide 19 of tumstatin can suppress HepG2 proliferation and induce HepG2 apoptosis as shown by $\mathrm{H} \& \mathrm{E}$ staining and $\mathrm{AO} / \mathrm{EB}$ staining. It was found that the ratio of $\mathrm{Bcl}-2 / \mathrm{Bax}$ decreased and the expression of $\mathrm{Bid}$ increased during apoptosis. Cytochrome $\mathrm{C}$ was released from the mitochondria into the cytoplasm and the mitochondrial membrane potential decreased. These data suggest that peptide 19 of tumstatin induces cell apoptosis by the mitochondrial pathway. Also, we observed that the levels of Fas and p53 were closely correlated with those of the Bcl-2 family. These results indicate that peptide 19 of tumstatin upregulates the expression of these two proteins. Our data indicate that peptide 19 of tumstatin induces a change in Bcl-2 family expression and the release of cytochrome $\mathrm{C}$ by upregulating the expression of p53 and Fas in HepG2 cells. These results helped confirm that mitochondria were at the core of the apoptosis induced by peptide 19 of tumstatin. This provides the theoretical basis for the future application of peptide 19 as a chemotherapeutic treatment for tumors.

Anti-tumor drugs aiming at suppressing angiogenesis have the advantages of a broad spectrum, low toxicity and no 
drug resistance. Therefore, they have become a research hotspot and have come to be used in clinics. It has been shown that drugs that suppress angiogenesis interfere with drug transport into solid tumors while inhibiting tumor growth. The reduced blood supply makes the tumor cell anoxic. This makes the tumor more easily acquire resistance to radio treatment and chemical treatment and produces genetic instability. By natural selection, tumor cells resistant to anoxia survive and others are killed. This leaves a more malignant tumor there and makes the therapy more complicated. Therefore, drugs that only suppress tumor angiogenesis must be used together with drugs inhibiting tumor cell proliferation in order to achieve the desired result. Understanding the mechanism of peptide 19 of tumstatin-induced tumor cell apoptosis is an important step forward. It should be possible to replace the chemotherapeutic agent with peptide 19 of tumstatin. There will be more effective anti-tumor activity and fewer toxic side effects when peptide 19 of tumstatin is combined with drugs that suppress angiogenesis.

\section{Acknowledgements}

This study was supported by the National Natural Science Foundation of China (No. 30472035).

\section{References}

1. Saus J, Wieslander J, Langeveld JP, Quinones S and Hudson BG: Identification of the Goodpasture antigen as the alpha 3 (IV) chain of collagen IV. J Biol Chem 263: 13374-13380, 1988.

2. Maeshima Y, Colorado PC, Torre A, et al: Distinct antitumor properties of a type IV collagen domain derived from basement membrane. J Biol Chem 275: 21340-21348, 2000.

3. Colorado PC, Torre A, Kamphaus G, et al: Anti-angiogenic cues from vascular basement membrane collagen. Cancer Res 60: 2520-2526, 2000.

4. Kamphaus GD, Colorado PC, Panka DJ, et al: Canstatin, a novel matrix-derived inhibitor of angiogenesis and tumor growth. J Biol Chem 275: 1209-1215, 2000.

5. Maeshima Y, Colorado PC and Kalluri R: Two RGD-independent alpha vbeta 3 integrin binding sites on tumstatin regulate distinct anti-tumor properties. J Biol Chem 275: 23745-23750, 2000 .
6. Petitclerc E, Boutaud A, Prestayko A, et al: New functions for non-collagenous domains of human collagen type IV. Novel integrin ligands inhibiting angiogenesis and tumor growth in vivo. J Biol Chem 275: 8051-8061, 2000.

7. Maeshima Y, Manfredi M, Reimer C, et al: Identification of the anti-angiogenic site within vascular basement membrane-derived tumstatin. J Biol Chem 276: 15240-15248, 2001.

8. Maeshima Y, Yerramalla UL, Dhanabal M, et al: Extracellular matrix-derived peptide binds to alpha(v)beta(3) integrin and inhibits angiogenesis. J Biol Chem 276: 31959-31968, 2001.

9. Maeshima Y, Sudhakar A, Lively JC, et al: Tumstatin, an endothelial cell-specific inhibitor of protein synthesis. Science 295: 140-143, 2002.

10. Floquet N, Pasco S, Ramont L, et al: The antitumor properties of the alpha3(IV)-(185-203) peptide from the NC1 domain of type IV collagen (tumstatin) are conformation-dependent. J Biol Chem 279: 2091-2100, 2004.

11. Wang SJ, Liu XH, JI YB and Chen N: Study on biological activity of two modified anti-tumor peptide of tumstatin. Prog Biochem Biophys 34: 1152-1161, 2007.

12. Desai BN, Myers BR and Schreiber SL: FKBP12-rapamycinassociated protein associates with mitochondria and senses osmotic stress via mitochondrial dysfunction. Proc Natl Acad Sci USA 99: 4319-4324, 2002.

13. Khor TO, Gul YA, Ithnin H and Seow HF: A comparative study of the expression of Wnt-1, WISP-1, survivin and cyclin-D1 in colorectal carcinoma. Int J Colorectal Dis 21: 291-300, 2006.

14. Li P, Nijhawan D, Budihardjo I, Srinivasula SM, Ahmad M, Alnemri ES and Wang X: Cytochrome c and dATP-dependent formation of Apaf-1/caspase-9 complex initiates an apoptotic protease cascade. Cell 91: 479-489, 1997.

15. Brenner C and Kroemer G: Apoptosis. Mitochondria - the death signal integrators. Science 289: 1150-1151, 2000.

16. Green DR and Reed JC: Mitochondria and apoptosis. Science 281: 1309-1312, 1998.

17. Tsujimoto Y and Shimizu S: Bcl-2 family: life-or-death switch. FEBS Lett 466: 6-10, 2000.

18. Zhang L, Yu J, Park BH, Kinzler KW and Vogelstein B: Role of BAX in the apoptotic response to anticancer agents. Science 290: 989-992, 2000.

19. Gross A, Jockel J, Wei MC and Korsmeyer SJ: Enforced dimerization of BAX results in its translocation, mitochondrial dysfunction and apoptosis. EMBO J 17: 3878-3885, 1998.

20. Sheikh MS and Fornace AJ Jr: Role of p53 family members in apoptosis. J Cell Physiol 182: 171-181, 2000.

21. Bennett M, Macdonald K, Chan SW, Luzio JP, Simari R and Weissberg P: Cell surface trafficking of Fas: a rapid mechanism of p53-mediated apoptosis. Science 282: 290-293, 1998. 